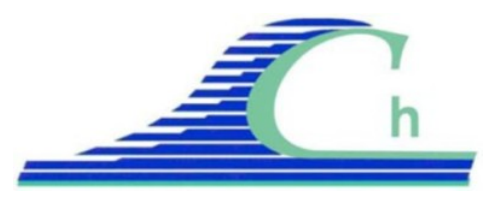

XII ${ }^{\text {èmes }}$ Journées Nationales Génie Côtier - Génie Civil

Cherbourg, 12-14 juin 2012

DOI:10.5150/jngcgc.2012.072-B @ Editions Paralia CFL

disponible en ligne - http://www.paralia.fr - available online

\title{
Rejointoiement des ouvrages en site maritime
}

\section{Nicolas BOURNETON ${ }^{1}$, Jacques BILLON ${ }^{1}$, Michel MENGUY ${ }^{2}$, Benoît THAUVIN ${ }^{2}$, Laurent RIOU ${ }^{2}$}

1. Cete de l'Ouest, Division Infrastructure et Environnement, Groupe Ouvrage d'Art, MAN, Rue René Viviani, BP 46223, 4426 Nantes cedex 2, France.

2. Cete de l'Ouest, Laboratoire Régional des Ponts et Chaussées de St-Brieuc, 5 rue Jules Vallès, 22015 Saint Brieuc Cedex, France. nicolas.bourneton@developpement-durable.gouv.fr

\section{Résumé :}

Le présent article traite du rejointoiement des ouvrages maritimes en maçonnerie (pierres ou briques). Après avoir rappelé le contexte des ouvrages en maçonnerie vieillissant, différentes techniques de régénération des structures sont présentées en précisant des critères de choix associés. Les différentes techniques de rejointoiement sont alors développées tout en précisant les caractéristiques nécessaires à imposer aux différents matériaux. Le contrôle d'exécution étant primordial, les éléments à vérifier au niveau des contrôles externes et internes est aussi fourni.

\section{Mots-clés :}

Génie côtier - Rejointoiement- Maçonnerie - Travaux maritimes - Travaux portuaires

\section{Introduction}

Le présent article rassemble les principaux éléments du guide "rejointoiement des ouvrages en maçonnerie" qui sera publié par le Cetmef (www.cetmef.developpementdurable.gouv.fr). Le lecteur se reportera à ce guide pour plus de précisions sur les points abordés ainsi que sur les études préalables et le phasage de chantier, ..., ou pour tout élément concernant le référentiel technique.

Dans le domaine maritime, les ouvrages en maçonnerie rencontrés sont principalement des quais, des jetées, des digues et des phares. Il n'est plus construit d'ouvrage en maçonnerie depuis environ la fin du XIX ${ }^{\text {ième }}$ siècle. Aujourd'hui, âgées, fatiguées, il n'est donc pas étonnant que ces structures posent quelques problèmes à de nombreux gestionnaires. Les joints et les pierres des maçonneries sont particulièrement sensibles aux actions des eaux : eaux de surface issues de la plate-forme, eaux internes circulant dans le corps des remblais, eaux de mer. Cette eau agit sur la structure soit par effet physique (érosion/abrasion, possible action du gel, ...), soit par effet chimique (dissolution des liants, ...).

Suivant ces dégradations, le rejointoiement permet de :

- rétablir la résistance mécanique des joints entre les pierres vis-à-vis du caractère agressif du milieu (chimiquement et/ou mécaniquement), 
- reconstituer le "monolithisme" originel de la maçonnerie pour assurer la résistance globale de la structure,

- éviter les circulations d'eau anarchiques entre moellons,

- préserver la "respiration" des pierres, c'est-à-dire l'ensemble des échanges thermohydro-chimiques qui ont lieu entre les pores de la pierre et l'air ambiant,

- restituer l'esthétique de l'ouvrage.

Le rejointoiement consiste à remplacer un joint dégradé voire détruit. Le rejointoiement ne concerne que le remplacement des joints et non celui du mortier de hourdage. Dans les cas extrêmes où la reconstitution du hourdage s'avère être nécessaire, il est fait appel à la technique de l'injection ou du matage pour les altérations peu profondes.

Il existe plusieurs formes de joints possibles. Les joints doivent être traités de telle sorte qu'ils permettent l'écoulement des eaux de ruissellement.

Il convient de proscrire les joints en saillies qui se brisent sous l'action du gel ou des mouvements de la structure ou sous d'éventuels chocs. De même, les joints creux trop profonds $(>1 \mathrm{~cm})$ sont à éviter, car ils retiennent l'eau et toutes sortes d'impuretés.

Il est nécessaire de procéder à un examen détaillé de l'ouvrage afin de dresser un inventaire complet des désordres. Il faut ensuite rechercher leurs causes, les mécanismes par lesquels ils se sont développés, puis inventorier leurs conséquences possibles sur la vie de l'ouvrage.

\section{Les différentes techniques de régénération de maçonnerie}

Le choix de la méthode de régénération dépend de différents paramètres. Le schéma donné en figure 1, définit les domaines d'application des différentes méthodes que l’on décrit par la suite.

Les méthodes de régénération dépendent :

- des moyens de mise en œuvre du mortier (méthode manuelle dite traditionnelle ou méthode mécanique, par projection, par injection),

- de l'influence sur la répartition des efforts entre les pierres (méthode passive qui fige l'état actuel ou méthode active qui s'accompagne d'une mise en charge des joints et des pierres avoisinantes, cas des joints matés),

Les critères de choix des méthodes concernent l'ouverture et la profondeur des joints. Pour l'ouverture des joints notée $\mathrm{O}$ on aura les possibilités suivantes :

- si $\mathrm{O}>15 \mathrm{~mm}$ : projection possible, si surface de joint est supérieure à $100 \mathrm{~m}^{2}$.

- si $\mathrm{O}>10 \mathrm{~mm}$ : joints matés possibles.

- si $\mathrm{O}<10 \mathrm{~mm}$ : fermeture de la surface puis injection.

Pour la profondeur des joints notée $P$ on retiendra que :

- si P>50 mm, le rejointoiement dit traditionnel n’est pas adapté,

- si P>200 mm, la projection n'est plus suffisante. Il faut utiliser l'injection en complément. 


\section{XII ${ }^{\text {èmes }}$ Journées Nationales Génie Côtier - Génie Civil \\ Cherbourg, 12-14 juin 2012}

Le matage peut être utilisé jusqu'à $\mathrm{P}=200 \mathrm{~mm}$ environ. C'est la technique à privilégier dans les petites réparations. S'il apparaît des trous de plus de $5 \mathrm{~cm}$ de largeur, il faudra procéder à l'ajout de moellons pour réduire les joints.

Le présent article ne traite que du rejointoiement, donc que du traitement des maçonneries sur une profondeur de 5 à $8 \mathrm{~cm}$ pour des ouvertures de $5 \mathrm{~mm}$ et plus.

La spécificité du milieu maritime peut influer sur le choix de la méthode. En effet, sur des zones difficiles d'accès ou très vite recouvertes par les eaux (travail à la marée) la méthode de traitement la plus rapidement mise en œuvre doit être privilégiée. Des problèmes de mise en œuvre (barges, nacelle, ...) sont à considérer dans un milieu particulièrement agité (batillage, houle, ...).

Inévitablement ces influences d'ordre technique ont des répercussions sur le critère économique des réparations.

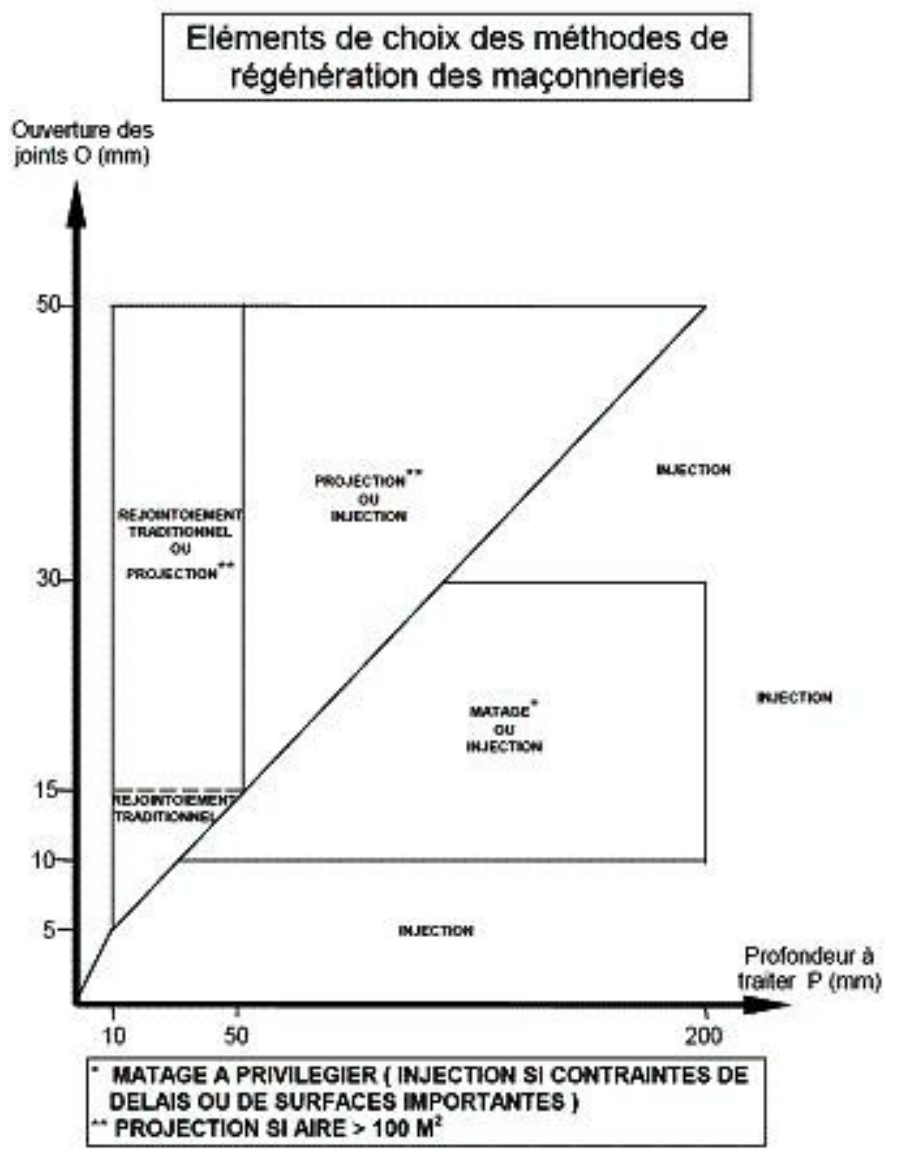

Figure 1 Eléments de choix des matériaux de régénération des maçonneries.

\section{Le choix des produits}

Pour ce type de travaux où le coût de la part de main d'œuvre est prédominante par rapport au coût des fournitures, on cherchera à utiliser des produits faciles à employer 
qui assurent un maximum de garantie de tenue dans le temps. Le recours à des mortiers "prêts à l'emploi" sera donc privilégié pour les zones de marnage ou d'accès difficiles. La formulation du mortier et le choix de chaque constituant sera fait en fonction : de l'exposition de la partie de l'ouvrage à traiter, de l'ouverture des joints, de la nature des pierres constituant la maçonnerie, de la technique de mise en œuvre choisie, de la durée entre mise en œuvre et immersion pour les zones de marnage et des contraintes esthétiques et environnementales.

\subsection{Techniques traditionnelle et matage}

La norme NF P95-107 "Réparation et renforcement des maçonneries" précise les caractéristiques des constituants du mortier. Les mortiers doivent être conformes à la norme NF EN 998-2 "Définitions et spécifications des mortiers pour maçonnerie". Les mortiers bâtards "chaux et ciment" seront à proscrire en raison des risques d'attaque de la chaux par l'eau de mer et en raison de leurs temps de prise très lents.

La compatibilité entre le mortier de rejointoiement et le mortier de hourdage sera vérifié par analyse chimique.

\subsection{Techniques par projection}

La norme NF P95-102 "Réparation et renforcement des ouvrages en béton et maçonnerie- béton projeté" précise les caractéristiques des constituants des bétons.

\section{3 $\underline{\text { Choix des constituants }}$}

\subsubsection{Ciments}

Les ciments employés devront être conformes aux normes: NF EN 197-1 pour les ciments courants, NF EN 197-2 relative à évaluation de la conformité, NF P15-307 pour les ciments à maçonner, NF $\mathrm{P}$ 15-317 pour les ciments destinés aux travaux à la mer (PM), NF EN 15-319 concernant les ciments pour travaux en eaux à haute teneur en sulfure (ES), P 18-011 pour les bétons et la classification des environnements agressifs. Pour le rejointoiement en site maritime, il faut se limiter aux seuls ciments compatibles "pour des travaux à la mer". L'entrepreneur doit proposer à l'acceptation du maître d'œuvre, dans le cadre de son Plan d'Assurance Qualité (PAQ) et conjointement avec les études des bétons, la catégorie, la classe, la sous-classe et la provenance des ciments devant être utilisés. L'emploi de ciments aux laitiers confère au liant une meilleure durabilité vis-à-vis de l'attaque marine, cependant avec ces ciments, des apparitions en surface de tâches inesthétiques sont possibles. Les liants doivent être choisis après analyse de la pierre ou de la brique en place afin d'éviter les attaques telles que l'expansion par les sulfates ou encore les attaques alcaline ou basique. 


\section{XII ${ }^{\text {èmes }}$ Journées Nationales Génie Côtier - Génie Civil \\ Cherbourg, 12-14 juin 2012}

\subsubsection{Sable}

Le sable choisi sera conforme aux normes : NF EN 12620, des granulats pour béton et NF P 18-545 relative aux granulats- éléments de définition, conformité et codification. Les granulats marins sont interdits. Le sable utilisé pour confectionner le mortier de rejointoiement est un sable de rivière lavé. Le sable utilisé aura une influence sur la couleur des joints. En cas d'exigences esthétiques particulières des planches d'essais sont à prévoir.

\subsubsection{Eau de gâchage}

L'eau de gâchage doit être conforme à NF EN 1008 pour l'eau de gâchage. Le gâchage à l'eau de mer est interdit. L'eau provenant du réseau public d'eau potable est réputée conforme à la norme.

\subsubsection{Produits "prêts à l'emploi"}

Les produits utilisés doivent être conformes à la norme NF EN 1504-3 et ils seront adaptés à l'environnement maritime.

Une prise rapide du produit facilitera la mise en œuvre dans les zones de marnage. A ce titre le PAQ déterminera la durée pratique d'utilisation du produit nécessaire et celle du produit retenu devra être supérieure.

\section{Le choix des techniques de rejointoiement}

Le rejointoiement doit être réalisé le plus vite possible après le dégarnissage. Pour cela il est fortement préconisé de prévoir des surfaces de travaux permettant le dégarnissage et le rejointoiement dans la même journée mais également en fonction des impératifs de chantier (marnage, ...).

\subsection{Le rejointoiement manuel par la méthode traditionnelle}

Au préalable, le joint est humidifié par aspersion et non par immersion massive. Le rejointoiement par la méthode traditionnelle se fait par jet manuel, avec bourrage à "la langue de chat" et refoulement au fer.

Il est recommandé de procéder de haut en bas pour réduire les salissures et de nettoyer les pierres adjacentes aux joints traités. En fin de poste de travail, une cure de la zone rejointoyée est à réaliser. Elle se fait par humidification, voire par aspersion, avec si nécessaire une protection contre le soleil et le vent.

La cure du parement rejointoyé permet de limiter le retrait des joints et leur dessiccation.

Dans le cas de traitement de zones marnantes, il devra être procédé de bas en haut pour permettre une meilleure prise du mortier par rapport aux conditions de marée. 
On doit veiller à placer les évents au travers des joints en cas d'injection ultérieure, à établir des joints dans le plan du parement et à ne pas réaliser le lissage des joints au fer qui entraînerait un ressuage de leur surface et la fragiliserait.

La finition des joints peut se faire avec une éponge ou une brosse après une "prise" suffisante du mortier. La surface obtenue présente un aspect légèrement granuleux. Le type de finition est défini en accord avec le maître d'œuvre en fonction des planches d'essai réalisées préalablement aux travaux.

\subsection{Le rejointoiement manuel par matage}

Le matage est réalisé dans des joints dégarnis en profondeur et permet une remise en charge partielle de la maçonnerie par reconstitution locale du mortier de hourdage.

Une première couche de mortier est disposée en fond de joint avec un matage modéré. Après séchage de la première couche, (les couches complémentaires sont réalisées par 2 cm d'épaisseur), chaque couche est matée énergiquement avant la mise en œuvre de la suivante en laissant, à l'approche du parement, la place pour réaliser un joint traditionnel de $4 \mathrm{~cm}$ de profondeur.

\subsection{Le rejointoiement par projection (voies sèche ou mouillée)}

\subsubsection{Projection par voie sèche}

Si l'on réalise le rejointoiement de maçonnerie par mortier projeté par voie sèche, le dosage en ciment conseillé est de $500 \mathrm{~kg} / \mathrm{m}^{3}$. Lors de la projection, l'extrémité de la buse est tenue à moins de $20 \mathrm{~cm}$ du parement et la force de débit est réglée pour que la projection atteigne le fond du joint à regarnir. Toutes les conditions de mise en œuvre sont consignées dans la norme NF P 95-102 aux articles 8 et 9.

\subsubsection{Projection par voie mouillée}

Si l'on réalise le rejointoiement de maçonnerie par mortier projeté par voie humide, le dosage en ciment conseillé est de $500 \mathrm{~kg} / \mathrm{m}^{3}$. La consistance du mortier est contrôlée par une mesure de l'affaissement au cône avant l'introduction du raidisseur (un affaissement de $12 \mathrm{~cm}$ sera couramment admis en flux dense). Lors de la projection, l'extrémité de la buse est tenue à moins de $10 \mathrm{~cm}$ du parement et la force de débit est réglée pour que la projection atteigne le fond du joint à regarnir. Toutes les conditions de mise en œuvre sont aussi consignées dans la norme NF P 95-102 articles 8 et 9.

Différents matériels d'application de rejointoiement par voie mouillée ont été comparés par le Cetmef, dans son rapport "Maintenance et réparation des ouvrages de génie civil en Mer. Ils concernent une pompe à vis, un pot sous pression et un pistolet pneumatique.

Le pistolet pneumatique a été conçu pour une autre application, il est à éviter. Les essais ont montré que les matériels "pompe à vis" et "pot sous pression" sont bien adaptés au 


\section{XII ${ }^{\text {èmes }}$ Journées Nationales Génie Côtier - Génie Civil \\ Cherbourg, 12-14 juin 2012}

rejointoiement. L'utilisation préférentielle d'un matériel est fonction des matériaux à appliquer.

\subsubsection{Choix entre voie sèche ou mouillée}

Ces deux techniques se rapprochent plus de la régénération interne que du rejointoiement simple car elles intéressent des profondeurs supérieures à $5 \mathrm{~cm}$. Ainsi la voie sèche permet un rejointoiement plus profond (20 à $25 \mathrm{~cm}$ par voie sèche pour au plus $15 \mathrm{~cm}$ par voie mouillée). Elle exige des joints plus larges (au moins $2 \mathrm{à} 3 \mathrm{~cm}$ ) que la voie mouillée. Elle est à privilégier lorsque l'on recherche une forte résistance mécanique et une forte adhérence. Mais la projection par voie sèche engendre une salissure des maçonneries qui peut être le plus souvent évitée par voie mouillée

\section{Les contrôles d'exécution}

En fonction de la technique utilisée et des produits mis en œuvre, les contrôles suivants doivent être effectués.

\subsection{Nettoyage}

Le nettoyage de la zone de travail doit faire l'objet d'une épreuve de convenance et être conforme aux dispositions prévues au Plan Qualité de l'entreprise, les exigences en termes de résultats et de moyens à mettre en œuvre seront vérifiées.

\subsection{Dégarnissage}

Le choix de la technique de dégarnissage est soumis à l'acceptation du maître d'œuvre et doit faire l'objet d'une épreuve de convenance afin d'apprécier son efficacité, sa maîtrise afin de préserver les pierres ou briques d'épaufrures éventuelles, sa profondeur du dégarnissage, sa consistance du fond de joint qui doit assurer un appui au mortier de rejointoiement et sa dureté du fond de joint qui est appréciée par enfoncement d'un poinçon.

\subsection{Ciments}

Conditions de livraison des ciments. Les conditions de livraison et de stockage des ciments doivent être conformes à l'article 3 du fascicule 3 des Cahiers des Clauses Techniques Générales.

En complément de celles-ci, le fournisseur doit informer des livraisons le maître d'œuvre au minimum 24 heures à l'avance.

Quant aux prélèvements conservatoires, l'entrepreneur doit effectuer selon les modalités prévues aux clauses 2.2. et 2.3. de la norme NF P 15-300 des prélèvements conservatoires de ciment à savoir $25 \mathrm{~kg}$ pour chaque lot de ciment utilisé pour les épreuves d'étude et de convenance des bétons, $5 \mathrm{~kg}$ pour chaque partie d'ouvrage définie 
lors de l'établissement du plan de contrôle d'exécution de l'ouvrage avec un prélèvement à la 1ère livraison de chaque ciment de qualité nouvelle.

Ces prélèvements sont conservés à l'abri en récipients étanches et étiquetés, soit par le laboratoire qui procédera aux analyses, soit par le maître d'œuvre. Des contrôles et essais sont à effectuer : pendant toute la durée des travaux, l'entrepreneur fournit au maître d'œuvre les relevés statistiques du fabricant de ciment concernant la moyenne, l'écart type, et le coefficient de variation.

Dans le cadre de son contrôle intérieur, l'entrepreneur doit se faire communiquer les résultats de l'autocontrôle effectué par la cimenterie sur le ciment livré et mettre ces résultats à la disposition du maître d'œuvre.

Pour le contrôle extérieur, le programme des prélèvements à effectuer est un prélèvement par partie d'ouvrage. Sur chaque prélèvement désigné par le maître d'œuvre, sont réalisés les essais de temps de prise et de flexion -compression à 7 et 28 jours-.

Dans le cas de résultats défavorables, il doit être procédé à des contre-épreuves dans les conditions du paragraphe 2.2.5. de la norme NF P 15-300. Pendant ces contre-épreuves, le maître d'œuvre peut faire bloquer le stock ou le silo concerné jusqu'à la conclusion de celles-ci.

Les résultats de ces essais doivent être communiqués au maître d'œuvre dans les soixante-douze heures qui suivent les prélèvements, et en tout état de cause avant l'emploi des ciments (excepté les essais de résistance).

Le reste des prélèvements de ciment après essais, est conservé durant 6 mois. Le maître d’œuvre se réserve la possibilité de modifier la cadence de ces essais.

\section{$5.4 \underline{\text { Sables }}$}

Le PAQ indique la provenance des granulats et le niveau de performance des granulats proposés. Il en fixe les modalités du contrôle du transport, du stockage et de la conformité des fournitures. Pendant toute la durée du chantier, le sable doit provenir d'une seule et même installation. Chaque proposition doit être obligatoirement accompagnée des fuseaux de production établis à partir d'un contrôle statistique à la production portant sur une durée d'au moins 6 mois. L'effectif de l'échantillon correspondant à chaque caractère spécifié ne doit pas être inférieur à 30.

En conformité avec la norme NF EN.196-1, le granulat à utiliser est un sable tamisé et lavé, de bonne qualité et d'origine alluvionnaire, de préférence EV>75.

Un sable de bonne qualité est constitué de grains allant de $0,08 \mathrm{~mm}$ à $0,5 \mathrm{~mm}$ suivant une courbe de granulométrie régulière. La proportion maximale d'éléments retenus sur le tamis de module 35, tamis de 2,5 mm doit être inférieure à 10\%. Des planches d'essai sont à réaliser avant rejointoiement pour juger de la couleur du joint à mettre en place. 